\title{
Iterative Learning Control Design and Application for Linear Continuous Systems with Variable Initial States Based on 2-D System Theory
}

\author{
Wei Guan, ${ }^{1}$ Qiao Zhu, ${ }^{2}$ Xu-Dong Wang, ${ }^{1}$ and Xu-Hui Liu ${ }^{1}$ \\ ${ }^{1}$ Beijing Institute of Control Engineering, Beijing 100190, China \\ ${ }^{2}$ School of Automation \& Electrical Engineering, University of Science and Technology Beijing, Beijing 100083, China \\ Correspondence should be addressed to Qiao Zhu; zhuqiao_ustb@163.com
}

Received 14 February 2014; Revised 22 April 2014; Accepted 12 May 2014; Published 22 May 2014

Academic Editor: Xinzhu Meng

Copyright (C) 2014 Wei Guan et al. This is an open access article distributed under the Creative Commons Attribution License, which permits unrestricted use, distribution, and reproduction in any medium, provided the original work is properly cited.

This paper is concerned with the variable initial states problem in iterative learning control (ILC) for linear continuous systems. Firstly, the properties of the trajectory of 2-D continuous-discrete Roesser model are analyzed by using Lyapunov's method. Then, for any variable initial states which absolutely converge to the desired initial state, some ILC design criteria in the form of linear matrix inequalities (LMI) are given to ensure the convergence of the PD-type ILC rules. The convergence for variable initial states implies that the ILC rules can be used to achieve the perfect tacking for variable initial states, even if the system dynamic is unknown. Finally, the micropropulsion system is considered to illustrate efficiency of the proposed ILC design criteria.

\section{Introduction}

During the past two decades, a great deal of attention has been paid to the ILC. This is a particularly interesting control algorithm since it can progressively improve the tracking performance of the systems by a learning mechanism using the information of errors and inputs in the preceding trials. Consequently, there have been a lot of ILC techniques presented in the area of control systems (see the books $[1,2]$, the recent survey paper [3], and references therein).

In general, a boundary condition named as identical initialization condition (i.i.c) is indispensable to achieve the perfect tracking performance of ILC rules. However, for many practical control systems, i.i.c is hardly achievable. Therefore, the variable initial states problem in ILC is frequently investigated. For example, in [4], for linear timevarying systems, an initial state learning algorithm has been proposed to achieve perfect tracking by making the initial state a convergent sequence, which requires the initial states are accessible and reachable. If the initial states are only accessible, an alternative is introduced in [5] for nonlinear systems by revising the target trajectory nearby the initial state into a new one. For a class of nonlinear systems with some different initial conditions, the convergence property of ILC was deeply investigated in [6]. Furthermore, if the system input matrix is accurately known, it is shown in [7] that the perfect tracking also can be achieved for any bounded initial tracking errors.

In recent years, the 2-D system theory was successfully and widely introduced to the ILC approach (see [8-13]). However, the i.i.c is still a fundamental assumption for the perfect tracking. For example, for a linear continuous system with i.i.c, the 2-D system theory based ILC method was presented in [8]. Furthermore, it is worth noting that Lyapunov stability theory of 2-D nonlinear systems is deeply studied under some special boundary conditions (see [1417]). It motivates the authors to relax the i.i.c of ILC for linear continuous systems by using 2-D system theory. This is the origin of this study.

The organization of this study is as follows. In next section, the convergence of the trajectory of 2-D continuousdiscrete Roesser model with respect to the discrete time index 
is investigated. In Section 3, some sufficient conditions in the form of LMI are derived to guarantee the convergence of PDtype ILC rules for any initial states which absolutely converge to the desired initial state. It implies that the perfect tracking can be fulfilled for variable initial states even if the system dynamics are unknown. In Section 4, the micropropulsion system is given to show the effectiveness of the results presented in the study.

Notations. Let $\mathbb{R}^{n}$ denote the $n$-dimensional Euclidean space, $\mathbb{R}^{n \times m}$ the set of all $n \times m$ real matrices, $\mathbb{R}_{+}^{n \times m}$ the set of all $n \times$ $m$ real matrices with nonnegative elements, $\mathbb{R}_{+}$the set of all nonnegative real numbers, and $\mathbb{Z}_{+}$the set of all nonnegative integers. $I_{n}$ denotes the $n \times n$ identity matrix, $\|\cdot\|$ the usual Euclidean norm, and $\rho(\cdot)$ the spectral radius. $\dot{\mathbf{x}}(t, k)$ denotes the partial derivative $\partial \mathbf{x}(t, k) / \partial t$ and “*” the elements below the main diagonal of a symmetric matrix.

\section{2-D Continuous-Discrete System Theory}

Consider the following 2-D continuous-discrete Roesser model:

$$
\left[\begin{array}{c}
\dot{\mathbf{x}}^{h}(t, k) \\
\mathbf{x}^{v}(t, k+1)
\end{array}\right]=\left[\begin{array}{ll}
\mathbf{A}_{11} & \mathbf{A}_{12} \\
\mathbf{A}_{21} & \mathbf{A}_{22}
\end{array}\right]\left[\begin{array}{l}
\mathbf{x}^{h}(t, k) \\
\mathbf{x}^{v}(t, k)
\end{array}\right],
$$

where $t \in[0, T], k \in \mathbb{Z}_{+} ; \mathbf{x}=\left[\begin{array}{l}\mathbf{x}^{h}(i, j) \\ \mathbf{x}^{v}(i, j)\end{array}\right] \in \mathbb{R}^{n}$ is the state, where $\mathbf{x}^{h} \in \mathbb{R}^{n_{1}}, \mathbf{x}^{v} \in \mathbb{R}^{n_{2}}\left(n_{1}+n_{2}=n\right)$ represent the horizontal and vertical states, respectively; $\mathfrak{A}=\left[\begin{array}{ll}\mathbf{A}_{11} & \mathbf{A}_{12} \\ \mathbf{A}_{21} & \mathbf{A}_{22}\end{array}\right]$ is the system matrix with the submatrices $A_{i j}, i, j=1,2$ of appropriate dimensions.

Lemma 1. For a time-invariant linear discrete system $\mathbf{x}(k+$ $1)=\mathbf{A x}(k)+\mathbf{B u}(k)$, the solution satisfies $\lim _{k \rightarrow \infty} \mathbf{x}(k)=0$, if $\rho(\mathbf{A})<1,\|\mathbf{x}(0)\|<\infty$ and $\sum_{k=0}^{\infty}\|\mathbf{u}(k)\|<\infty$.

Proof. It is easy to see that $x(k)=A^{k} x(0)+\sum_{i=0}^{k-1} A^{i} B u(k-1-i)$. Note that there exist real constants $\beta>0$ and $0<\gamma<1$ such that $\left\|A^{k}\right\| \leq \beta \gamma^{k}$ if $\rho(A)<1$. So it follows that

$$
\|x(k)\| \leq \beta \gamma^{k}\|x(0)\|+\beta\|B\| \sum_{i=0}^{k-1} \gamma^{i}\|u(k-1-i)\| .
$$

Note that

$$
\begin{aligned}
\sum_{k=0}^{\infty} & \sum_{i=0}^{k-1} \gamma^{i}\|u(k-1-i)\| \\
& =\sum_{i=0}^{\infty} \gamma^{i} \sum_{k=i+1}^{\infty}\|u(k-1-i)\| \\
& =\sum_{i=0}^{\infty} \gamma^{i} \sum_{k=0}^{\infty}\|u(k)\|=\frac{1}{1-\gamma} \sum_{k=0}^{\infty}\|u(k)\|<\infty .
\end{aligned}
$$

Then, we have $\sum_{i=0}^{k-1} \gamma^{i}\|u(k-1-i)\| \rightarrow 0$ as $k \rightarrow \infty$. Thus, we can easily complete the proof from (2).

Our goal in the section is the following.

Theorem 2. Suppose 2-D continuous-discrete system (1) satisfy boundary condition

$$
\begin{gathered}
\left\|\mathbf{x}^{v}(t, 0)\right\| d t<\infty, \quad \forall t \in[0, T], \\
\sum_{k=0}^{\infty}\left\|\mathbf{x}^{h}(0, k)\right\|<\infty .
\end{gathered}
$$

Then, the 2-D system trajectory satisfies

$$
\lim _{k \rightarrow \infty} \mathbf{x}(t, k)=0, \quad \forall t \in[0, T]
$$

if there exist symmetric positive definite matrices $\mathbf{W}_{1} \in \mathbb{R}^{n_{1} \times n_{1}}$ and $\mathbf{W}_{2} \in \mathbb{R}^{n_{2} \times n_{2}}$ such that

$$
\left[\begin{array}{cc}
\Sigma_{11} & \Sigma_{12} \\
* & A_{22}^{T} \mathbf{W}_{2} A_{22}-\mathbf{W}_{2}
\end{array}\right]<0,
$$

where $\boldsymbol{\Sigma}_{11}=\mathbf{A}_{11}^{T} \mathbf{W}_{1}+\mathbf{W}_{1} \mathbf{A}_{11}+\mathbf{A}_{21}^{T} \mathbf{W}_{2} \mathbf{A}_{21}, \boldsymbol{\Sigma}_{12}=\mathbf{W}_{1} \mathbf{A}_{12}+$ $\mathbf{A}_{21}^{T} \mathbf{W}_{2} \mathbf{A}_{22}$.

Proof. From (1), we have $\mathbf{x}^{v}(t, k+1)=\mathbf{A}_{22} \mathbf{x}^{v}(t, k)+\mathbf{A}_{21} \mathbf{x}^{h}(t, k)$. It implies from Lemma 1 that $\lim _{k \rightarrow \infty} \mathbf{x}^{v}(t, k)=0$ if $\rho\left(\mathbf{A}_{22}\right)<$ 1 and $\sum_{k=0}^{\infty}\left\|\mathbf{x}^{h}(t, k)\right\|<\infty$.

Now, let us prove $\sum_{k=0}^{\infty}\left\|\mathbf{x}^{h}(t, k)\right\|<\infty$ from the inequality $\dot{V}_{1}\left(\mathbf{x}^{h}(t, k)\right)+V_{2}\left(\mathbf{x}^{v}(t, k+1)\right)-V_{2}\left(\mathbf{x}^{v}(t, k)\right)<0$ which is equivalent to the inequality (6), where $V_{1}(\mathbf{x})=\mathbf{x}^{T} \mathbf{W}_{1} \mathbf{x}, V_{2}(\mathbf{x})=$ $\mathbf{x}^{T} \mathbf{W}_{2} \mathbf{x}$. Clearly, from the inequality $\dot{V}_{1}\left(\mathbf{x}^{h}(t, k)\right)+V_{2}\left(\mathbf{x}^{v}(t, k+\right.$ 1)) $-V_{2}\left(\mathbf{x}^{v}(t, k)\right)<0$, we have

$$
\begin{aligned}
\sum_{k=0}^{\infty} \dot{V}_{1}\left(\mathbf{x}^{h}(t, k)\right) & \\
< & \sum_{k=0}^{\infty} V_{2}\left(\mathbf{x}^{v}(t, k)\right) \\
& -\sum_{k=0}^{\infty} V_{2}\left(\mathbf{x}^{v}(t, k+1)\right) \leq V_{2}\left(\mathbf{x}^{v}(t, 0)\right),
\end{aligned}
$$

which implies

$$
\sum_{k=0}^{\infty} V_{1}\left(\mathbf{x}^{h}(t, k)\right)
$$

$$
\begin{aligned}
< & \sum_{k=0}^{\infty} V_{1}\left(\mathbf{x}^{h}(0, k)\right) \\
& +\int_{0}^{t} V_{2}\left(\mathbf{x}^{v}(\tau, 0)\right) d \tau \\
\leq & \sum_{k=0}^{\infty} V_{1}\left(\mathbf{x}^{h}(0, k)\right) \\
& +\int_{0}^{T} V_{2}\left(\mathbf{x}^{v}(t, 0)\right) d t .
\end{aligned}
$$


It follows that $\sum_{k=0}^{\infty} V_{1}\left(\mathbf{x}^{h}(t, k)\right)<\infty, \sum_{k=0}^{\infty}\left\|\mathbf{x}^{h}(t, k)\right\|<\infty$ and $\lim _{k \rightarrow \infty} \mathbf{x}^{h}(t, k)=0$ if the boundary condition (4) and the inequality (6) hold. Note that the inequality (6) implies $\rho\left(\mathbf{A}_{22}\right)<1$. Therefore, the proof is completed.

\section{2-D Iterative Learning Algorithm}

Consider the following time-invariant linear continuous system

$$
\begin{gathered}
\dot{\mathbf{x}}(t, k)=\mathbf{A} \mathbf{x}(t, k)+\mathbf{B u}(t, k), \\
\mathbf{y}(t, k)=\mathbf{C} \mathbf{x}(t, k),
\end{gathered}
$$

where $t \in[0, T]$ is the continuous-time index, $k \in[0, \infty)$ is the iteration index; $\mathbf{x}(t, k) \in \mathbb{R}^{n}, u(t, k) \in \mathbb{R}^{m}$, and $\mathbf{y}(t, k) \in$ $\mathbb{R}^{p}$ are the state, input, and output, respectively; $\mathbf{A} \in \mathbb{R}^{n \times n}$, $\mathbf{B} \in \mathbb{R}^{n \times m}$, and $\mathbf{C} \in \mathbb{R}^{p \times n}$ are real matrices. An ILC rule is given as

$$
\mathbf{u}(t, k+1)=\mathbf{u}(t, k)+\Delta \mathbf{u}(t, k)
$$

where $\Delta \mathbf{u}$ is the modification of input that will be designed later. Then, one can now introduce the following definition.

Definition 3. A learning rule (10) is said to be convergent if it generates a sequence $\{\mathbf{u}(t, k)\}$ for system (9) such that $\mathbf{y}(t, k) \rightarrow \mathbf{y}_{r}(t)$ as $k \rightarrow \infty$ for any $t \in[0, T]$, where $\mathbf{y}_{r}(t)$ is a derivable reference trajectory given in advance.

Let us denote $\mathbf{e}(t, k)=\dot{\mathbf{y}}_{r}(t)-\dot{\mathbf{y}}(t, k), \boldsymbol{\eta}(t, k)=\mathbf{x}(t, k+$ $1)-\mathbf{x}(t, k)$. Then, we have $\dot{\boldsymbol{\eta}}(t, k)=\dot{\mathbf{x}}(t, k+1)-\dot{\mathbf{x}}(t, k)=$ $\mathbf{A}[\mathbf{x}(t, k+1)-\mathbf{x}(t, k)]+\mathbf{B}[\mathbf{u}(t, k+1)-\mathbf{u}(t, k)]=\mathbf{A} \boldsymbol{\eta}(t, k)+$ $\mathbf{B} \Delta \mathbf{u}(t, k)$ and $\mathbf{e}(t, k+1)-\mathbf{e}(t, k)=-\mathbf{C}[\dot{\mathbf{x}}(t, k+1)-\dot{\mathbf{x}}(t, k)]=$ $-\mathbf{C A} \boldsymbol{\eta}(t, k)-\mathbf{C B} \Delta \mathbf{u}(t, k)$. Thus, the 2-D control error system can be presented in the form of 2-D continuous-discrete Roesser model:

$$
\left[\begin{array}{c}
\dot{\boldsymbol{\eta}}(t, k) \\
\mathbf{e}(t, k+1)
\end{array}\right]=\left[\begin{array}{cc}
\mathbf{A} & 0 \\
-\mathbf{C A} & \mathbf{I}_{p}
\end{array}\right]\left[\begin{array}{l}
\boldsymbol{\eta}(t, k) \\
\mathbf{e}(t, k)
\end{array}\right]+\left[\begin{array}{c}
\mathbf{B} \\
-\mathbf{C B}
\end{array}\right] \Delta \mathbf{u}(t, k) .
$$

Now, let us consider the boundary conditions. From the boundary condition (4) of 2-D system (1), the boundary condition of the 2-D system (9)-(10) can be assumed to be absolutely convergent; that is,

$$
\sum_{k=0}^{\infty}\left\|\mathbf{x}(0, k)-\mathbf{x}_{r}(0)\right\|<\infty, \quad \mathbf{C x}_{r}(0)=\mathbf{y}_{r}(0) .
$$

It implies that $\sum_{k=0}^{\infty}\|\boldsymbol{\eta}(0, k)\|<\infty$ and $\lim _{k \rightarrow \infty} \mathbf{y}(0, k)=$ $\mathbf{y}_{r}(0)$. Without loss of generality, the initial input can be chosen as zero; that is, $\mathbf{u}(t, 0)=0, \forall t \in[0, T]$. Furthermore, it is obvious that $\|\mathbf{e}(t, 0)\| \leq\left\|\dot{\mathbf{y}}_{r}(t)\right\|+\|\mathbf{C A}\|\|\mathbf{x}(t, 0)\|<\infty$. Thus, the initial states of $2-\mathrm{D}$ error system (11) satisfy the boundary condition (4). It implies that Theorem 2 can be used to analyze the convergence of 2-D systems (9)-(10).

Theorem 4. Suppose 2-D systems (9) and (10) satisfy the boundary condition (12). If there exist symmetric positive definite matrices $\mathbf{P}_{1} \in \mathbb{R}^{n \times n}, \mathbf{P}_{2} \in \mathbb{R}^{p \times p}$ and real matrices $\mathbf{Q}_{1} \in \mathbb{R}^{m \times n}, \mathbf{Q}_{2} \in \mathbb{R}^{m \times p}$ such that the following $L M I$

$$
\left[\begin{array}{ccc}
\mathbf{\Pi}_{11} & \mathbf{B Q}_{2} & -\left(\mathbf{C A P}_{1}+\mathbf{C B Q}_{1}\right)^{T} \\
* & -\mathbf{P}_{2} & \mathbf{P}_{2}-\left(\mathbf{C B Q}_{2}\right)^{T} \\
* & * & -\mathbf{P}_{2}
\end{array}\right]<0,
$$

holds, where $\mathbf{\Pi}_{11}=\mathbf{P}_{1} \mathbf{A}^{T}+\left(\mathbf{B} \mathbf{Q}_{1}\right)^{T}+\mathbf{A P}_{1}+\mathbf{B} \mathbf{Q}_{1}$, then the PD-type ILC rule

$$
\begin{aligned}
\mathbf{u}(t, k+1)= & \mathbf{u}(t, k)+\mathbf{K}_{1} \boldsymbol{\eta}(t, k)+\mathbf{K}_{2} \mathbf{e}(t, k) \\
= & \mathbf{u}(t, k)+\mathbf{K}_{1}[\mathbf{x}(t, k+1)-\mathbf{x}(t, k)] \\
& +\mathbf{K}_{2}\left[\dot{\mathbf{y}}_{r}(t)-\dot{\mathbf{y}}(t, k)\right],
\end{aligned}
$$

is convergent with $\mathbf{K}_{1}=\mathbf{Q}_{1} \mathbf{P}_{1}^{-1}, \mathbf{K}_{2}=\mathbf{Q}_{2} \mathbf{P}_{2}^{-1}$.

Proof. It is not hard to see that the system matrix of 2-D error system (11) with ILC rule (14) is

$$
\left[\begin{array}{cc}
\mathbf{A}+\mathbf{B K}_{1} & \mathbf{B K}_{2} \\
-\mathbf{C A}-\mathbf{C B K}_{1} & \mathbf{I}_{p}-\mathbf{C B K}_{2}
\end{array}\right]
$$

From Theorem 2, the asymptotic stability of 2-D error system (11) with ILC rule (14) can be guaranteed by the following inequality:

$$
\left[\begin{array}{cc}
\mathbf{F}_{11} & \mathbf{F}_{12} \\
* & -\mathbf{W}_{2}
\end{array}\right]+\left[\begin{array}{c}
\widetilde{\mathbf{F}}_{13} \\
\widetilde{\mathbf{F}}_{23}
\end{array}\right] \mathbf{W}_{2}\left[\begin{array}{c}
\widetilde{\mathbf{F}}_{13} \\
\widetilde{\mathbf{F}}_{23}
\end{array}\right]^{T}<0,
$$

where $\mathbf{F}_{11}=\left(\mathbf{A}+\mathbf{B} \mathbf{K}_{1}\right)^{T} \mathbf{W}_{1}+\mathbf{W}_{1}\left(\mathbf{A}+\mathbf{B} \mathbf{K}_{1}\right), \mathbf{F}_{12}=$ $\mathbf{W}_{1} \mathbf{B} K_{2}, \widetilde{\mathbf{F}}_{13}=-\left(\mathbf{C A}+\mathbf{C B K}_{1}\right)^{T}, \widetilde{\mathbf{F}}_{23}=\left(\mathbf{I}_{p}-\mathbf{C B K}_{2}\right)^{T}$; here $\mathbf{W}_{1} \in \mathbb{R}^{n \times n}, \mathbf{W}_{2} \in \mathbb{R}^{p \times p}$ are symmetric positive definite matrices. By using the well-known Schur complement lemma, the inequality (16) can be rewritten as

$$
\left[\begin{array}{ccc}
\mathbf{F}_{11} & \mathbf{F}_{12} & \mathbf{F}_{13} \\
* & -\mathbf{W}_{2} & \mathbf{F}_{23} \\
* & * & -\mathbf{W}_{2}
\end{array}\right]<0,
$$

where $\mathbf{F}_{13}=-(\mathbf{C A}+\mathbf{C B K})^{T} \mathbf{W}_{2}, \mathbf{F}_{23}=\left(\mathbf{I}_{p}-\mathbf{C B K}\right)^{T} \mathbf{W}_{2}$. Pre- and postmultiplying the above inequality by the matrix $\operatorname{diag}\left\{\mathbf{W}_{1}^{-1}, \mathbf{W}_{2}^{-1}, \mathbf{W}_{2}^{-1}\right\}$, we have

$$
\left[\begin{array}{ccc}
\mathbf{G}_{11} & \mathbf{G}_{12} & \mathbf{G}_{13} \\
* & -\mathbf{W}_{2}^{-1} & \mathbf{G}_{23} \\
* & * & -\mathbf{W}_{2}^{-1}
\end{array}\right]<0,
$$

where $\mathbf{G}_{11}=\mathbf{W}_{1}^{-1}\left(\mathbf{A}+\mathbf{B} \mathbf{K}_{1}\right)^{T}, \mathbf{G}_{12}=\mathbf{B} \mathbf{K}_{2} \mathbf{W}_{2}^{-1}, \mathbf{G}_{13}=$ $-\mathbf{W}_{1}^{-1}(\mathbf{C A}+\mathbf{C B K})^{T}, \mathbf{G}_{23}=\mathbf{W}_{2}^{-1}\left(\mathbf{I}_{p}-\mathbf{C B K}\right)^{T}$. Let $\mathbf{P}_{1}=$ $\mathbf{W}_{1}^{-1}, \mathbf{P}_{2}=\mathbf{W}_{2}^{-1}, \mathbf{Q}_{1}=\mathbf{K}_{1} \mathbf{P}_{1}, \mathbf{Q}_{2}=\mathbf{K}_{2} \mathbf{P}_{2}$. Then, it is not hard to show that the inequality (18) can be rewritten as the LMI (13). It implies that the LMI (13) guarantees $\lim _{k \rightarrow \infty} \dot{\mathbf{y}}(t, k)=\dot{\mathbf{y}}_{r}(t), \forall t \in[0, T]$. Note that the boundary condition (12) implies $\lim _{k \rightarrow \infty} \mathbf{y}(0, k)=\mathbf{y}_{r}(0)$. Then, we have $\lim _{k \rightarrow \infty} \mathbf{y}(t, k)=\mathbf{y}_{r}(t), \forall t \in[0, T]$. Therefore, the proof is completed. 
From Theorem 4, we can easily obtain the following theorem.

Theorem 5. Suppose 2-D systems (9) and (10) satisfy the boundary condition (12). If there exist symmetric positive definite matrices $\mathbf{P}_{1} \in \mathbb{R}^{n \times n}, \mathbf{P}_{2} \in \mathbb{R}^{p \times p}$ and real matrices $\mathbf{Q}_{2} \in \mathbb{R}^{m \times p}$ such that the following $L M I$

$$
\left[\begin{array}{ccc}
\mathbf{P}_{1} \mathbf{A}^{T}+\mathbf{A P}_{1} & \mathbf{B Q}_{2} & \left.-(\mathbf{C A P})_{1}\right)^{T} \\
* & -\mathbf{P}_{2} & \mathbf{P}_{2}-\left(\mathbf{C B Q}_{2}\right)^{T} \\
* & * & -\mathbf{P}_{2}
\end{array}\right]<0,
$$

holds, then the ILC rule

$$
\begin{aligned}
\mathbf{u}(t, k+1) & =\mathbf{u}(t, k)+\mathbf{K e}(t, k) \\
& =\mathbf{u}(t, k)+\mathbf{K}\left[\dot{\mathbf{y}}_{r}(t)-\dot{\mathbf{y}}(t, k)\right],
\end{aligned}
$$

is convergent with $\mathbf{K}=\mathbf{Q}_{2} \mathbf{P}_{2}^{-1}$.

Furthermore, the boundary condition (12) can be further relaxed if the system dynamic is known; that is, the system matrices $A, B, C$ are accurately known.

Theorem 6. Suppose the system matrices $A, B, C$ are accurately known with $\operatorname{rank}(\mathbf{C B})=\operatorname{rand}(\mathbf{C})=q$ and the initial states satisfy

$$
\lim _{k \rightarrow \infty} \mathbf{x}(0, k)=\mathbf{x}_{r}(0)
$$

Let $\mathbf{K}_{2}^{*}=(\mathbf{C B})^{T}\left[\mathbf{C B}(\mathbf{C B})^{T}\right]^{-1}$ and $\mathbf{K}_{1}^{*}=-\mathbf{K}_{2}^{*} \mathbf{C A}$. Then, the ILC rule

$$
\begin{aligned}
\mathbf{u}(t, k+1)= & \mathbf{u}(t, k)+\mathbf{K}_{1}^{*} \boldsymbol{\eta}(t, k)+\mathbf{K}_{2}^{*} \mathbf{e}(t, k) \\
= & \mathbf{u}(t, k)+\mathbf{K}_{1}^{*}[\mathbf{x}(t, k+1)-\mathbf{x}(t, k)] \\
& +\mathbf{K}_{2}^{*}\left[\dot{\mathbf{y}}_{r}(t)-\dot{\mathbf{y}}(t, k)\right],
\end{aligned}
$$

is convergent.

Proof. If $\operatorname{rank}(\mathbf{C B})=\operatorname{rand}(\mathbf{C})=q$, then $\mathbf{I}_{p}-\mathbf{C B K}_{2}=$ 0 and $-\mathbf{C A}-\mathbf{C B K}_{1}=0$ have unique solutions $\mathbf{K}_{2}^{*}=$ $(\mathbf{C B})^{T}\left[\mathbf{C B}(\mathbf{C B})^{T}\right]^{-1}$ and $\mathbf{K}_{1}^{*}=-\mathbf{K}_{2}^{*} \mathbf{C A}$. Clearly, from the 2-D error system (11), it is not hard to see that $\mathbf{e}(t, k)=0, \forall k \geq 1$, $t \in[0, T]$ for any initial states $\boldsymbol{\eta}(0, k)$ and $e(t, 0)$. If follows that $\lim _{k \rightarrow \infty} \dot{\mathbf{y}}(t, k)=\dot{\mathbf{y}}_{r}(t), \forall t \in[0, T]$. From the boundary condition $\lim _{k \rightarrow \infty} \mathbf{x}(0, k)=\mathbf{x}_{r}(0)$, the theorem can be easily proved.

Remark 7. Theorems 4-6 can be considered as extensions of the results in [8]. The construction method of 2-D error system (9) is different with that in [8], which makes us be able to analyze the variable initial states. From Theorems 4 and 5, we can see that the PD-type ILC rules can be used to achieve the perfect output tracking for linear continuous systems with any variable initial states which absolutely converge to the desired initial state, even if the system dynamics are unknown. Furthermore, the absolutely convergent boundary condition (12) can be relaxed to be convergent, but the system dynamic must be accurately known (see Theorem 6).

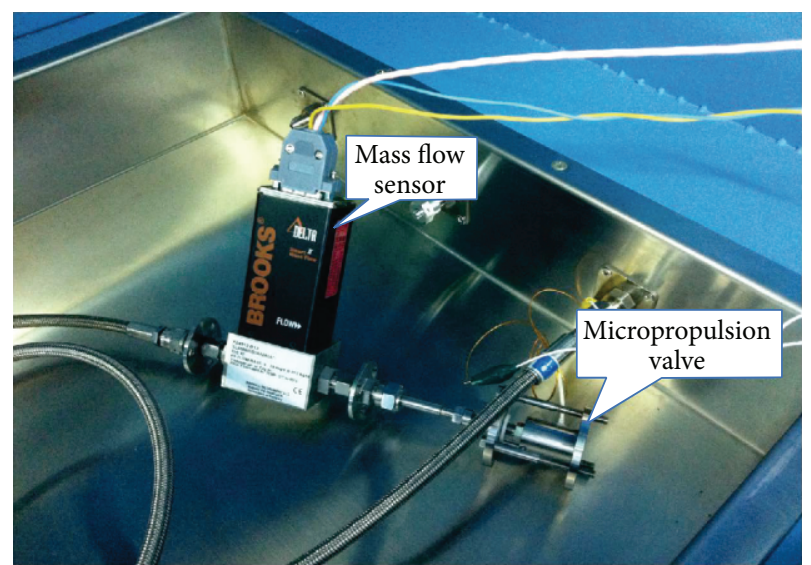

FIgURE 1: Photo of micropropulsion experimental system.

\section{Application to Micropropulsion System}

To illustrate the efficiency of the proposed ILC design criteria, we consider the micropropulsion system shown in Figure 1. The thrust of the micropropulsion system can be continuously controlled by adjusting the current. Since the thrust is difficult to be real-time detected, the mass flow rate is measured and fed back. Meanwhile, the relationship between mass flow rate and thrust will be demarcated by experiments.

As we know, the micropropulsion system is a classic nonlinear system but could be approximated as a linear system in the current interval $[70,120] \mathrm{mA}$. In this interval, the dynamical model of the micropropulsion system could be presented as

$$
G(s)=\frac{Y(s)}{U(s)}=\frac{0.446}{1+0.05 s},
$$

which can be realized in state-space model (9) with $A=20$, $B=8.92, C=1$, where $y=x$ is the mass flow rate $(\mathrm{mg} / \mathrm{s})$ and $u$ is the current (mA).

In the field of aerospace and medicine, the micropropulsion systems are sometimes required to repetitively output thrust or mass flow. As such, assume the admired mass flow rate $y_{r}(t)=4 \sin (5 t)+12(\mathrm{mg} / \mathrm{s}), t \in[0,2]$ and the initial states $x(0, k)=0.9^{k}+12$. By solving the LMI (13), we obtain the PD-type ILC law (14) with $K_{1}=-2.28, K_{2}=0.06$. Under the PD-type ILC law, the convergence profiles of the total square error $\mathscr{E}(k)=\int_{t=0}^{T}\left|y_{r}(t)-y_{k}(t)\right| d t$ for simulation and actual operation along the iteration axis $k$ are demonstrated in Figure 2.

\section{Conclusions}

The main contribution of this study is to show that the perfect tracking for linear continuous systems by ILC can be achieved for variable initial states which absolutely converge to desired initial state, even if the system dynamics are unknown. In order to show this result, the convergence of the trajectory of 2-D continuous-discrete Roesser model is investigated by using Lyapunov's method. The efficiency of the proposed 


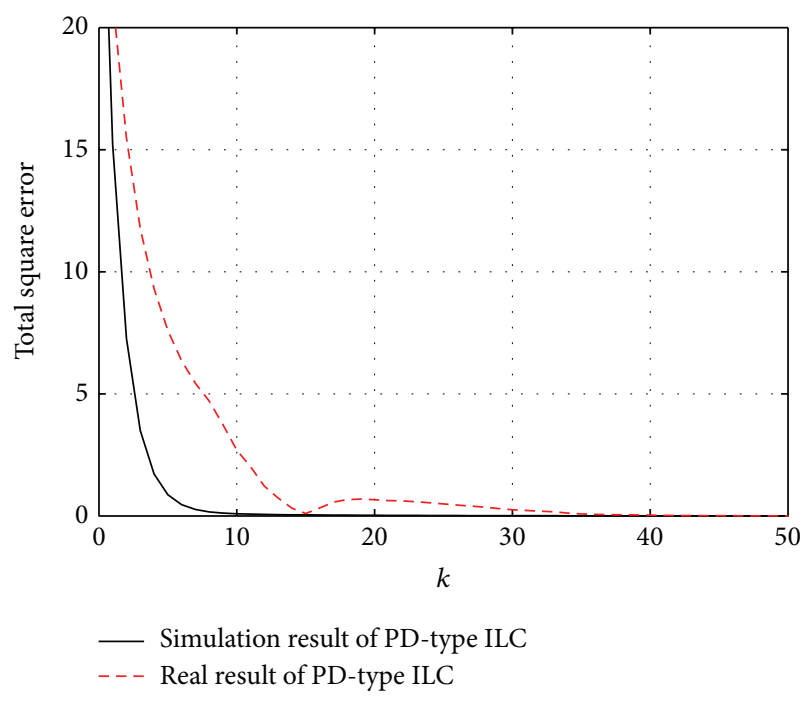

FIGURE 2: Simulation and actual operation results of the micropropulsion system along the iteration axis.

ILC design criteria is demonstrated by the micropropulsion system. Furthermore, it is well known that the 2-D systems are closely associated with the ILC and partial differential equations (see [18]). By studying the asymptotic stability of 2-D systems with proper boundary conditions, interesting results may be obtained for ILC and partial differential equations and others.

\section{Conflict of Interests}

The authors declare that there is no conflict of interests regarding the publication of this paper.

\section{Acknowledgments}

This work was supported by the National Natural Science Foundation of China (nos.61304087, 61333002, and 11371053), National High-tech R\&D Program of China (863 Program) (2013AA040705), Beijing Municipal Natural Science Foundation (no. 4132065), and Beijing Higher Education Young Elite Teacher Project (no. YETP0374).

\section{References}

[1] J.-X. Xu and Y. Tan, Linear and Nonlinear Iterative Learning Control, vol. 291 of Lecture Notes in Control and Information Sciences, Springer, Berlin, Germany, 2003.

[2] J.-X. Xu, S. K. Panda, and T. H. Lee, Real-Time Iterative Learning Control, Springer, London, UK, 2009.

[3] J.-X. Xu, "A survey on iterative learning control for nonlinear systems," International Journal of Control, vol. 84, no. 7, pp. 1275-1294, 2011.

[4] Y. Chen, C. Wen, Z. Gong, and M. Sun, "An iterative learning controller with initial state learning," IEEE Transactions on Automatic Control, vol. 44, no. 2, pp. 371-376, 1999.

[5] M. Sun and D. Wang, "Iterative learning control with initial rectifying action," Automatica, vol. 38, no. 8, pp. 1177-1182, 2002.
[6] J.-X. Xu and R. Yan, "On initial conditions in iterative learning control," IEEE Transactions on Automatic Control, vol. 50, no. 9, pp. 1349-1354, 2005.

[7] X.-D. Li, T. W. S. Chow, J. K. L. Ho, and J. Zhang, "Iterative learning control with initial rectifying action for nonlinear continuous systems," IET Control Theory \& Applications, vol. 3, no. 1, pp. 49-55, 2009.

[8] T. Chow and Y. Fang, "An iterative learning control method for continuous-time systems based on 2-D system theory," IEEE Transactions on Circuits and Systems I: Fundamental Theory and Applications, vol. 45, no. 6, pp. 683-689, 1998.

[9] J. E. Kurek and M. B. Zaremba, "Iterative learning control synthesis based on 2-D system theory," IEEE Transactions on Automatic Control, vol. 38, no. 1, pp. 121-125, 1993.

[10] X. Li, J. Ho, and T. Chow, "Iterative learning control for linear time-variant discrete systems based on 2-D systems theory," IEE Proceedings: Control Theory and Applications, vol. 152, no. 1, pp. 13-18, 2005.

[11] D. Meng, Y. Jia, J. Du, and S. Yuan, "Robust discrete-time iterative learning control for nonlinear systems with varying initial state shifts," IEEE Transactions on Automatic Control, vol. 54, no. 11, pp. 2626-2631, 2009.

[12] X.-D. Li, T. W. S. Chow, and J. K. L. Ho, "2-D system theory based iterative learning control for linear continuous systems with time delays," IEEE Transactions on Circuits and Systems I: Regular Papers, vol. 52, no. 7, pp. 1421-1430, 2005.

[13] S. S. Saab, "A discrete-time learning control algorithm for a class of linear time-invariant systems," IEEE Transactions on Automatic Control, vol. 40, no. 6, pp. 1138-1142, 1995.

[14] J. E. Kurek, "Stability of nonlinear parameter-varying digital 2D systems," IEEE Transactions on Automatic Control, vol. 40, no. 8, pp. 1428-1432, 1995.

[15] D. Liu, "Lyapunov stability of two-dimensional digital filters with overflow nonlinearities," IEEE Transactions on Circuits and Systems I: Fundamental Theory and Applications, vol. 45, no. 5, pp. 574-577, 1998.

[16] Q. Zhu and G.-D. Hu, "Stability and absolute stability of a general 2-D non-linear FM second model," IET Control Theory \& Applications, vol. 5, no. 1, pp. 239-246, 2011.

[17] Q. Zhu, G. D. Hu, and Y. X. Yin, "Lyapunov-type theorem of general 2-D nonlinear parameter-varying FM second model," IEEE Transactions on Circuits and Systems II, vol. 59, no. 7, pp. 453-457, 2012.

[18] W. Marszałek, "Two-dimensional state-space discrete models for hyperbolic partial differential equations," Applied Mathematical Modelling, vol. 8, no. 1, pp. 11-14, 1984. 


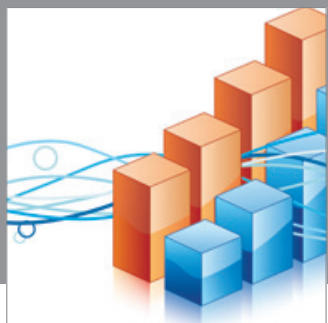

Advances in

Operations Research

mansans

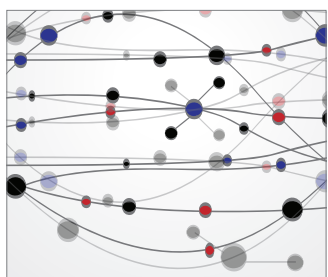

The Scientific World Journal
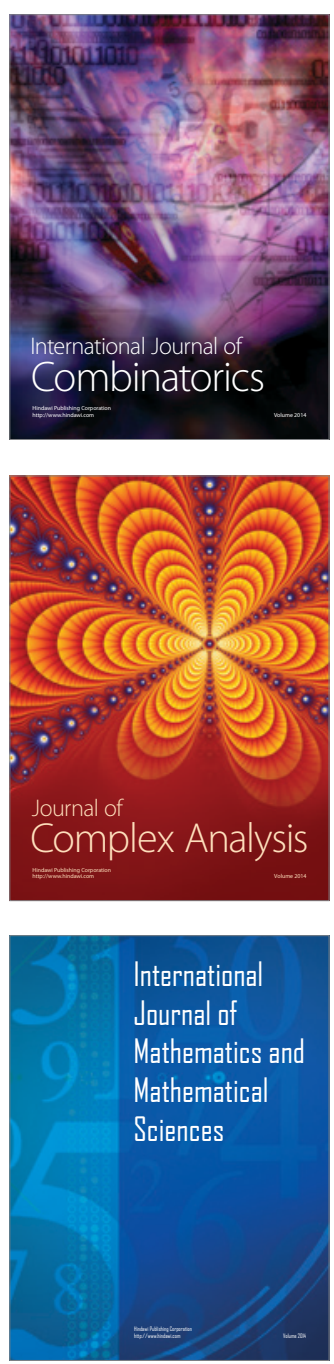
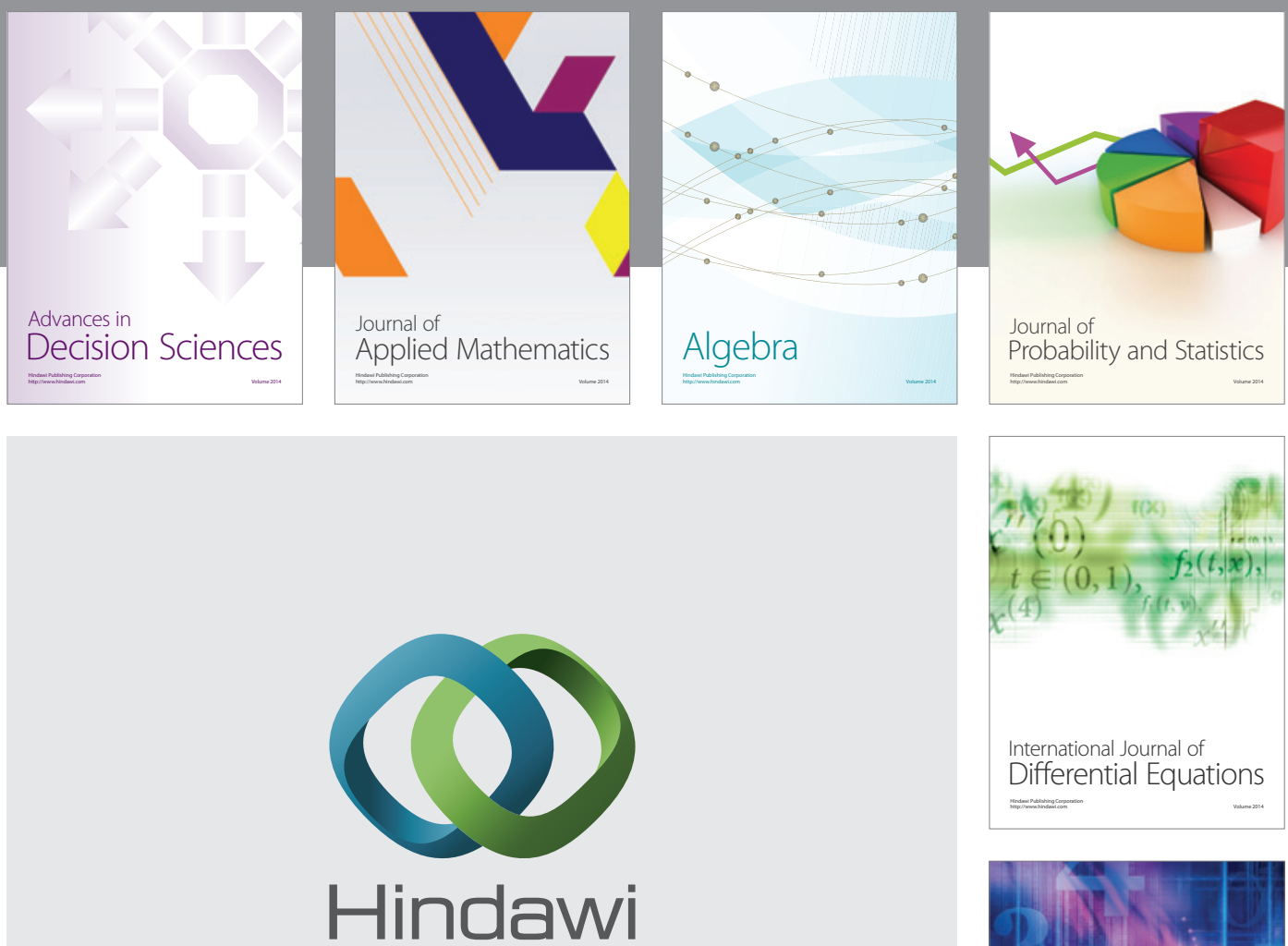

Submit your manuscripts at http://www.hindawi.com
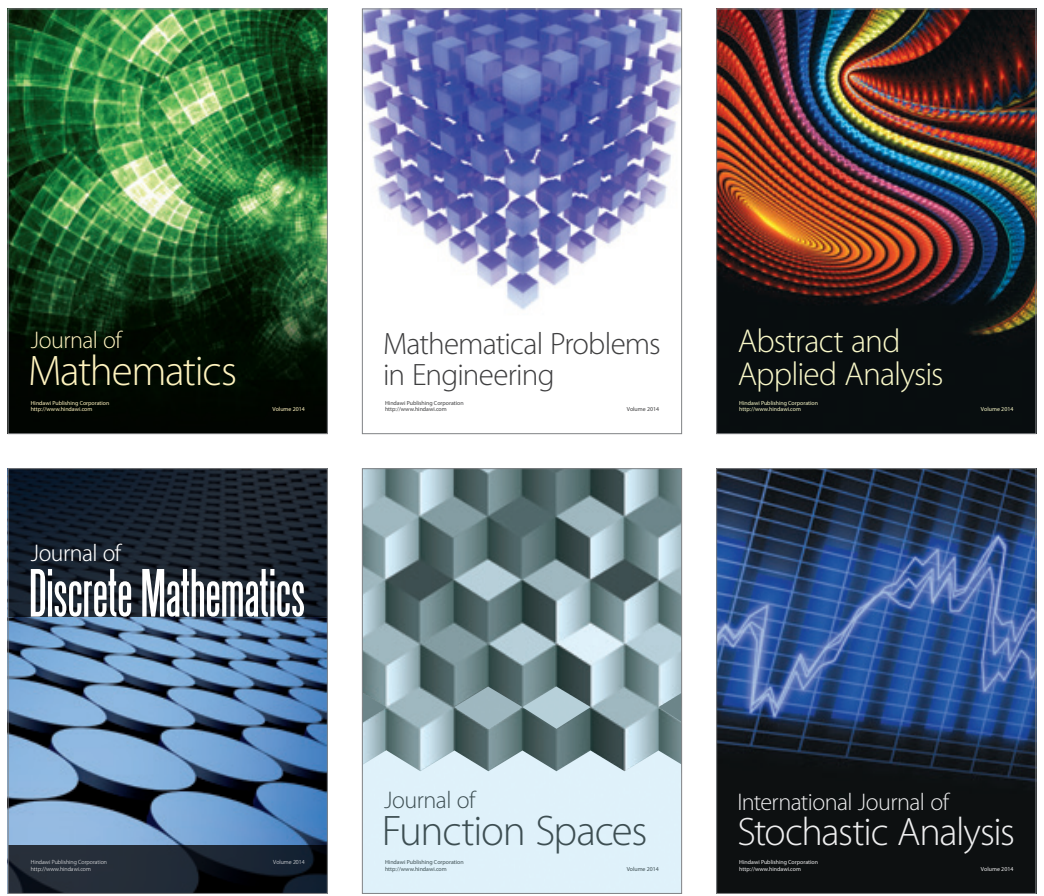

Journal of

Function Spaces

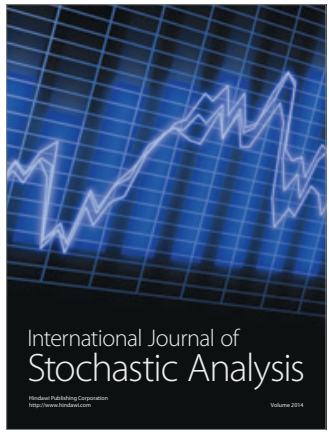

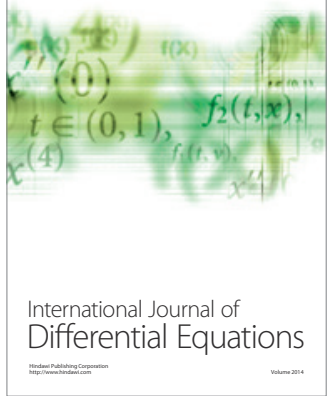
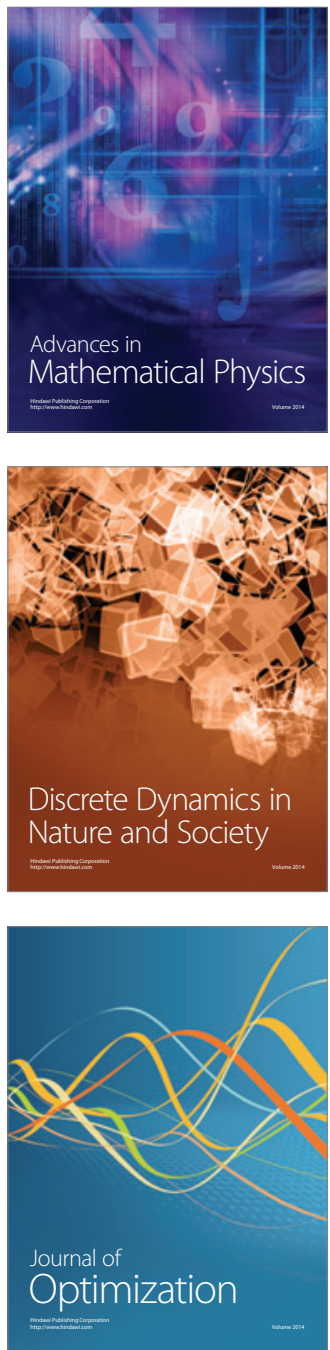